\title{
Tangence
}

\section{L'écrivain franco-ontarien entre le fantasme et le mythe}

\section{Lucie Hotte}

Numéro 56, décembre 1997

Postures scripturaires dans la littérature franco-ontarienne

URI : https://id.erudit.org/iderudit/025956ar

DOI : https://doi.org/10.7202/025956ar

Aller au sommaire du numéro

Éditeur(s)

Tangence

ISSN

0226-9554 (imprimé)

1710-0305 (numérique)

Découvrir la revue

Citer cet article

Hotte, L. (1997). L'écrivain franco-ontarien entre le fantasme et le mythe.

Tangence, (56), 26-39. https://doi.org/10.7202/025956ar d'utilisation que vous pouvez consulter en ligne.

https://apropos.erudit.org/fr/usagers/politique-dutilisation/ 


\title{
L'écrivain franco-ontarien entre le fantasme et le mythe
}

\author{
Lucie Hotte
}

et je réalise soudainement que tellement de poètes ont été tués par l'idée et/ou le mythe de la vie de poète

Patrice Desbiens, Sudbury

La littérature franco-ontarienne s'est inscrite d'emblée sous le signe de la prise de parole. Témoin la création des éditions Prise de parole, dont le nom, source de nombreuses hésitations, a finalement été choisi en raison des connotations qui pouvaient y être rattachées. Gaston Tremblay rappelle que le concensus s'est fait parce -qu'il y avait, dans ce nom, un glissement qui s'opérait entre écouter, recevoir, entendre et prendre la parole.'. L'existence de la maison d'édition trouvait à être validée par son nom: - Comme son nom le laisse entendre, la nouvelle maison croit que ce qui n'est pas exprimé n'existe pas et elle veut favoriser la prise de parole littéraire des Franco-Ontariens. ${ }^{2}$. Si prendre la parole, c'est s'octroyer le droit de parler, c'est aussi prendre part à un processus qui met en relation un locuteur, un discours et un récepteur. Quiconque prend la parole, en littérature, se situe par rapport à ceux et celles qui l'ont précédé, soit pour s'inscrire dans leur sillage, soit pour les dépasser, soit pour s'opposer à ce qu'ils représentent ou à ce qu'ils ont accompli. Selon Roland Barthes, ce qui s'impose d'abord à l'aspirant écrivain ce n'est pas la forme ou le contenu du discours existant mais bien la figure du locuteur, de l'écrivain. Vouloir prendre la parole en littérature, c'est d'abord vouloir s'instituer écrivain, c'est-à-dire exister ou agir de façon à correspondre à l'image mentale que l'on se fait de l'écrivain, ou encore - quoique Barthes n'envisage pas cette possibilité - s'y opposer. Cette image de l'écrivain qui préexiste à la prise de parole littéraire et qui l'oriente nécessairement, Roland Barthes l'appelle le fantasme d'écrivain: le fantasme d'écrivain

1 Gaston Tremblay, Prendre la parole. Le journal de bord du Grand CANO, Ottawa, Le Nordir, 1996, p. 71.

2 Ibid. 
régirait l'écriture même, en imposant à qui veut écrire une conception de l'écrivain, une posture scripturaire plutôt que le modèle d'un art.' Le fantasme d'écrivain, "c'est l'écrivain moins son cuvre " ${ }^{3}$. D'où, selon Jean-Louis Major, : [l]'écrivain comme fantasme, c'est une pose, une posture: une figure mythique ${ }^{4}$.

Si le fantasme d'écrivain apparaît comme éminemment subjectif, chez Barthes, dans la mesure où chaque aspirant écrivain aura en principe son propre fantasme, il n'en demeure pas moins que le fantasme d'écrivain, en tant que figure mythique, prend sa source dans l'imaginaire collectif. Selon Stanley Fish ${ }^{5}$, le lecteur ne reconnaît un poème que parce qu'il le construit en poème, au cours de l'acte de lecture, en fonction des caractéristiques qui sont conventionnellement associées à la poésie. De même, l'écrivain n'atteint son statut d'écrivain que par l'élaboration d'une corrélation entre sa vie et son ouvre d'une part et, d'autre part, un ensemble de présupposés ou de préjugés à l'égard de l'écrivain. On ne peut fantasmer l'écrivain qu'en fonction des écrivains que l'on connaît, que l'on reconnaît comme écrivain. La mythification de l'écrivain passe essentiellement par le discours que l'on tient sur lui et sur son œuvre. Si le fantasme d'écrivain suscite une posture scripturaire, il peut aussi imposer une posture lecturale ${ }^{6}$, qui ne viserait qu'à rechercher l'écrivain dans l'œuvre, qu'à prouver que l'écrivain existe et, par le fait même, qu'une littérature existe.

Paradoxalement (étant donné le nombre restreint d'écrivains), les littératures minoritaires sont, plus que les autres, marquées par le fantasme d'écrivain. Dans la mesure où l'existence d'une littérature apparait fragile ou menacée, les écrivains et les lecteurs, surtout les critiques, cherchent à en manifester ou démontrer l'existence à tout prix. Selon François Paré: "Plus les conditions d'oppression sont grandes dans l'histoire, plus la figure de

3 Roland Barthes, Roland Bartbes par Roland Barthes, Paris, Seuil, coll. "Écrivains de toujours*, 1975, p. 82.

4 Jean-Louis Major, - Perspectives esthétiques: fantasme d'écrivain et figure du texte •, Crémazte et Nelligan, Montréal, Fides, 1980, p. 169.

5 Stanley Fish, - How To Recognize a Poem When You See One ", Is There a Text in This Class?, Cambridge, Harvard University Press, 1980, p. 322-337.

6 - Lectural . se distingue de - lectoral *, dans la mesure où ce dernier terme renvoie à ce qui concerne le lecteur, alors que - lectural - désigne ce qui concerne la lecture. Voir Richard Saint-Gelais, Châteaux de pages. La fiction au risque de sa lecture, lasalle, Hurtubise HMH, coll. *Bèches -, 1994, 299 p. 
l'écrivain paraît garante de l'expression. . ${ }^{7}$ Garante de l'expression certes, mais surtout garante de l'existence de la littérature et, par là même, de l'existence de la communauté. Combien d'écrivains québécois ont-ils été les héritiers de Lord Durham, cherchant à prouver que celui-ci avait tort, qu'il existait bien une littérature d'ici, justifiant ainsi en retour leur propre existence ou leur propre définition fantasmatique!

La dynamique propre au fantasme d'écrivain trouve éminemment à s'accomplir, mieux qu'ailleurs, en milieu minoritaire. Prenons pour exemple un a écrivain - dont l'œuvre a marqué l'histoire littéraire de l'Ontario français: André Paiement, qui a été et est encore aujourd'hui considéré comme la figure de proue de la littérature franco-ontarienne. Il aurait été le catalyseur de toute l'activité créatrice d'une génération. Sa contribution à l'avènement d'une culture et d'une littérature franco-ontariennes est largement reconnue par la critique, qui lui accorde une place de choix dans le panthéon littéraire franco-ontarien. Comme le remarque François Paré, "[s]on œuvre et l'influence considérable qu'elle a eue et qu'elle continue d'avoir en Ontario français témoignent autant de l'originalité du projet littéraire en cause que de la force singulière de Paiement lui-même " ${ }^{8}$. À mon avis, l'œuvre de Paiement et la lecture qu'on en a faite témoignent tout autant ou davantage de l'emprise du fantasme d'écrivain sur la littérature franco-ontarienne.

\section{Posture scripturaire}

André Paiement arrive sur la scène littéraire de l'Ontario français à l'époque où il n'existe pas encore de littérature francoontarienne. Non pas qu'on n'ait jamais écrit en français en Ontario auparavant, mais bien plutôt parce que personne n'avait encore revendiqué le statut d'écrivain franco-ontarien, parce que personne avant lui ne parlait de littérature franco-ontarienne ni ne parlait en son nom. Or, s'ériger écrivain dans un contexte où les écrivains sont ceux qui écrivent ailleurs n'est pas tâche facile. Deux voies s'avèrent alors possibles: adopter la posture scripturaire de l'écrivain d'ailleurs ou refuser de correspondre à cette

7 François Paré, Théories de la fragtlité, Ottawa, Le Nordir,1994, p.135.

8 lbid., p. 66. 
image étrangère afin de manifester son originalité propre, c'est-àdire son origine propre. Paiement choisira cette seconde option, frayant ainsi la voie à tout un courant littéraire ${ }^{9}$ en Ontario français.

François Paré rappelle que * Paiement refusait la fixité du texte écrit et privilégiait des formes d'interventions orales sur le réel sociopolitique, interventions d'autant plus prégnantes qu'elles étaient évanescentes " ${ }^{10}$. Aussi ne s'est-il “ jamais préoccupé d'assurer à son œuvre une quelconque permanence en tant qu'ouvre littéraire" "1. Le caractère oral du texte viserait ainsi *à faire oublier l'écrit et, au-delà de cette critique de l'écrit, à mettre en doute la présence de l'écrivain en tant qu'écrivain. ${ }^{12}$. Faut-il pour autant croire que Paiement refusait de faire ouvre littéraire parce que, faute de modèles, il ne pouvait entretenir un fantasme d'écrivain? Au contraire, le fantasme d'écrivain était chez lui si. prégnant qu'il se devait de s'ériger contre lui. Si Paiement refuse l'écrivain en tant qu'écrivain, c'est qu'il a une conception du rôle de l'écrivain, de la posture propre à l'écrivain, et qu'il refuse de s'y conformer.

Selon Claude Abastado, l'écrivain en tant qu'écrivain serait modelé par le mythe romantique du Poète, al'écrivain par excellence "13. Parmi les nombreuses variantes du mythe, deux caractéristiques principales, opposées mais complémentaires, s'imposent : l'Écrivain est à la fois sacré et maudit. Il apparaît chez Hugo comme le "Mage ", chez Baudelaire sous le symbole du "phare *, chez Vigny sous la figure de "Moïse ", comme un prophète et un Messie. Deux traits le caractérisent alors: sa voyance et sa fonction messianique. Mais si le Mage possède le don de voir les choses telles qu'elles sont, s'il peut même montrer le chemin de l'avenir, il n'en est pas pour autant l'enfant chéri du peuple. "Les Mages sont le plus souvent solitaires et persécutés. "14 L'écrivain

9 Ce courant littéraire, François Paré le nomme celui de la conscience, alors que Robert Yergeau parlera de surcontextualisation. Voir François Paré, Les littératures de l'exiguité, Ottawa, Le Nordir, 1992, p. 123-135; Robert Ycrgeau, :Comment habiter le territoire fictionnel franco-ontarien?", Liaison, $\mathrm{n}^{\circ} 85$, janvier 1996, p. 30-32.

10 François Paré, Théories de la fragilité, op. cit., p. 67.

11 Ibid., p. 66.

12 Ibid., p. 70.

13 Claude Abastado, Mythes et rituels de l'écriture, Bruxelles, Éditions Complexe, coll. *Creusets-, 1979 , p. 61.

14 Ibid., p. 77. 
est alors perçu comme un prophète rejeté, inadapté à la vie "réelle ", incompris de ses contemporains, "exilé sur le sol au milieu des huées. ${ }^{15}$. C'est le "poète maudit "incarné par Baudelaire, Verlaine et Rimbaud ou, au Québec, par Nelligan, le fou, ou Saint-Denys Garneau, le martyr ${ }^{16}$. Devant choisir entre l'isolement ou la communion humaine, le Poète as'enferme dans une solitude dédaigneuse ou douleureuse, ou bien il assume une mission civilisatrice, sans pourtant jamais se mêler à ceux qu'il éclaire " ${ }^{17}$. Selon Claude Abastado, "[c]es représentations, contradictoires en apparence, fantasment en fait la même situation historique et elles ont le même sens; mais au niveau idéologique, elles reflètent les hésitations d'un choix politique et éthique. ${ }^{18}$.

Ainsi, tout concourt dans le mythe du Poète, à faire de l'Écrivain un être à part, en marge de la société, qui ne peut ni l'accepter, ni le comprendre. Pour André Paiement, dont le choix politique et éthique était de donner une portée sociale à son ouvre, il était impossible de devenir Écrivain. Il fallait à tout prix qu'il soit compris et accepté de la communauté franco-ontarienne. À cette vocation communautaire s'ajoutait l'exigence de se démarquer de la production littéraire québécoise, et surtout française, afin de produire une littérature plus authentique, plus nourricière. Ces deux contraintes l'engageaient donc à rejeter la *Littérature et à adopter une forme particulière de discours.

D'abord, le refus de l'écrivain lui impose un genre. Jean-Louis Major note que *[l]e fantasme d'écrivain est lié à une mythologie des genres littéraires, qui à son tour commande telle pose, telle posture ${ }^{19}$. Si la poésie apparaît comme la quintessence de la Littérature ${ }^{20}$, le théâtre, au contraire, est la forme la moins littéraire,

15 Charles Baudelaire, Les fleurs du mal, Paris, le livre de Poche, 1972, p. 180.

16 Voir la première partie *1. Le Mythe- de l'étude de Jean-Louis Major, "SaintDenys Gameau *, Le jeu en étoile, Ottawa, Éditions de l'Université d'Ottawa, 1978, p.83-100.

17 Claude Abastado, op. cit., p. 65.

18 Ibid., p. 65.

19 Jean-louis Major, - Perspectives esthétiques: fantasme d'écrivain et figure du texte ", op. cit., p. 169.

20 Une analyse de la place qu'occupe la poésie dans les littératures et du travail poétique qui cherche à transgresser les normes poétiques permettrait de souligner la force de la mythologie des genres littéraires. Les travaux d'Elisabeth lasserre sur l'ouvre de Patrice Desbiens illustrent ce rejet de la climension esthétique institutionnalisée du littéraire. 
dans la mesure où il n'est pas perçu comme écrit car il ne vise pas à être lu: la lecture du théâtre serait même, en quelque sorte, une perversion ${ }^{21}$. Le théâtre est, en somme, pure représentation: il est pour ainsi dire impossible d'y déceler la marque de l'auteur. Selon Northrop Frye, "l'absence de l'auteur, dissimulé à son auditoire, est un trait caractéristique du thềâtre " ${ }^{22}$. Mais dissimulé ne signifie pas pour autant inexistant: dans le théâtre, c'est l'origine qui demeure "illocalisable, d'où la possibilité pour l'Écrivain de devenir invisible. Si lauteur est absent, le destinataire est, en revanche, éminemment présent. La forme théâtrale, que retient Paiement, permet d'établir un contact direct avec le public. La présence du destinataire fait du théâtre une forme de rassemblement collectif apte à répondre aux exigences sociales de Paiement. Ainsi, le dramaturge se trouve éclipsé par les spectateurs.

De plus, l'œuvre de Paiement tire son origine de la création collective, où la notion même d'écrivain, et, par conséquent, celle de propriété intellectuelle, est absente. Michel Vaïs affirme que la création collective serait * néle] d'un rejet du texte-carcan et d'un refus de l'autorité omnisciente de l'écrivain. ${ }^{23}$. Mais elle est aussi, et peut-être surtout, le refus de s'arroger le pouvoir de dire. La création collective se présente comme un acte démocratique qui accorde à chacun le droit de parole. Cependant, il convient de signaler que cette méthode de travail a été dans une certaine mesure imposée à André Paiement. La décision de monter une création collective avait été prise par Pierre Bélanger, le directeur de la troupe universitaire à laquelle Paiement s'est joint en 1970. De plus, il ne s'agissait peut-être pas autant de création collective que de création individuelle. Gaston Tremblay rappelle le rôle primordial d'André Paiement dans l'écriture de cette première pièce, Moé j'viens du Nord s'tie!:

21 Jcan-Marie Schaeffer dans Qu'est-ce qu'un genne littëraire (Paris, Seuil, . Pó̀tique., 1989, p. 91) souligne le statut communicationnel ambigu du texte dramaticue, qui peut donner lieu à deux types de lecture: - Ce texte peut en effet, lorsquion le lit, avoir deux référents tout à fait distincts: si on le lit comme texte littéraire son référent est, comme celui d'une narration, une réalité historicjue ou imaginaire; en revanche, si on le lit comme texte théâtral, donc comme notation graphique d'une performance, son référent est la représentation théâtrale (bien entendu, lorsqu'il est représenté son référent est identique à celui qui est posé dans sa lecture comme texte littéraire).

22 Northrop Fryc, Anatomie de la critique, Paris, Gallimard, 1969, p. 302.

23 Michal Vaïs, L'écrivain scénique, Montréal, Les Presses de l'Université du Québec, 1978 , p. 240. 
Nous étions supposés écrire la pièce ensemble, en création collective. André Paiement détenait le rôle principal et il écrivit son propre monologue. Il nous est arrivé, le soir où nous étions censés l'aider à travailler, avec son dialogue complètement dactylographié. Deux semaines plus tard, après plusieurs nuits blanches, il avait écrit toute la pièce. ${ }^{24}$

Néanmoins, le fait que Paiement continuera à travailler surtout dans le contexte de la création collective et la place prépondérante que celle-ci prendra dans le théâtre franco-ontarien témoignent de l'importance de la valeur symbolique ${ }^{25}$ associée à cette méthode de travail.

De la même façon, le caractère oral de l'œuvre de Paiement trouve son origine dans le désir de communion avec la communauté. Mais le théâtre de Paiement est doublement oral. D'abord, l'oralité se manifeste dans le fait que les pièces sont axées vers la représentation plutôt que l'écriture, mais surtout, l'oralité émane d'un choix particulier de vocabulaire et de structures syntaxiques, propre à ce que Paiement appelle . le joual franco-ontarien 26 . Dans le liminaire de Lavalléville, Paiement souligne qu'*[i]l est très important que le texte de la pièce soit adapté à l'accent de la langue (quel qu'il soit) de la région où cette pièce est jouée "27. Il y a dans cette valorisation de la langue orale à la fois un refus de la sacralisation du langage littéraire et une affirmation d'appartenance. Utiliser le langage propre à un groupe, c'est affirmer: je suis comme vous, je fais aussi partie de ce groupe. La langue orale permet un rapport d'identification qui est par ailleurs sollicité par la thématique même des œuvres: celles-ci visent à représenter de façon réaliste le vécu des membres de la communauté. François Paré a sûrement raison lorsqu'il affirme que le refus d'être écrivain permet à André Paiement "de puiser directement, [...] aux richesses de la parole collective. ${ }^{28}$ et assure ainsi son

24 Gaston Tremblay, op. cit., p. 27.

25 Les * Notes du metteur en scène * qui précèdent le texte de La parole et la loi témoignent de l'absence, voulue, de la figure de l'auteur et du rejet de la littérature dans la création collective: - Le texte qui vous est présenté ici n'est ni la pièce d'un dramaturge, ni une œuvre de littérature. C'est le produit d'un travail théâtral ", La parole et la lot, Sudbury, Prise de parole, 1980, p. 6.

26 André Paiement, Lavalléville, Sudbury, Prise de parole, coll. *'Théâtre *, 1983 , p. 4.

27 lbid.

28 François Paré, Théories de la fragtlité, op. cit., p. 70. 
efficacité dans la communauté. Ainsi le fantasme d'écrivain qui anime Paiement l'amène à adopter la posture de l'anti-écrivain qui n'écrit que pour donner voix à sa communauté, pour témoigner de sa réalité et pour lui indiquer la voie à suivre.

\section{Le rapport à la collectivité}

Tout fantasme d'écrivain conjugue la représentation de l'écrivain au milieu social dans lequel il vit. L'écrivain comme fantasme est non seulement un sous-produit de la Littérature, il est également un sous-produit de la société. Encensé ou décrié, sacré ou maudit, ces épithètes lui seront accolées en fonction de la perception que l'on a du rôle qu'il peut jouer dans une société : en somme, de sa arentabilité . Ce n'est donc pas tant la figure de l'écrivain qui change que les connotations qui s'y rattachent. Si, dans certains contextes sociaux, l'écrivain peut apparaître comme une source de connaissance, soit parce qu'il manie les mots, soit parce qu'on le croit animé par une force occulte qui l'inspire, ailleurs il sera l'emblème de l'esthétisme, de l'art, de la beauté ou encore il sera perçu comme un être inutile, un parasite qui ne peut contribuer à l'économie de la nation. Ainsi, le contexte prescrit à l'écrivain, en partie du moins, sa place et son rôle dans la société. C'est dire que la posture scripturaire adoptée est le résultat de la conjonction d'une figure d'écrivain et d'un contexte socio-culturel. Comme le souligne Pierre Bourdieu :

le travail par lequel l'artiste fait son œuvre et se fait, inséparablement, comme artiste (et, lorsque cela fait partie de la demande du champ, comme artiste original, singulier) peut être décrit comme la relation dialectique entre son poste qui, souvent, lui préexiste et lui survit (avec des obligations, par exemple la -vie d'artiste -, des attributs, des traditions, des modes d'expresion, etc.) et son habitus qui le prédispose plus ou moins totalement à occuper ce poste ou - ce qui peut être un des préréquisits inscrits dans le poste - à le transformer plus ou moins complètement. ${ }^{29}$

Ce phénomène est éminemment visible dans la prise de position littéraire d'André Paiement. D'une part, il est confronté à la figure de l'écrivain élitiste, intellectuel, au-dessus du commun des

29 Pierre Bourdicu, "Mais qui a créé les créateurs?", Questions de scriologice, Paris, Minuit, 1980, p. 210. 
34

mortels, dont le discours est largement incompréhensible. D'autre part, il est membre d'une communauté composée principalement d'ouvriers, de mineurs et d'agriculteurs, souvent analphabètes, minoritaires, opprimés par la majorité anglophone, où l'écrivain n'a pas de place. L'image de l'écrivain qui trouve sa source dans l'histoire littéraire française est essentiellement étrangère à la réalité franco-ontarienne. Deux voies s'ouvrent alors à l'écrivain: écrire pour transformer le milieu ou pour contrer le contexte social en adoptant de plain-pied la posture de l'écrivain et en exploitant l'écart pour souligner le caractère maudit de l'écrivain, ou bien refuser d'être écrivain pour prendre racine dans le milieu. Plus s'accentuera l'écart entre les marques institutionnalisées de la figure de l'écrivain et le contexte social où il évolue, plus le contexte social contribuera à imposer une posture scripturaire orthodoxe ou révolutionnaire. Comme le dit Pierre Bourdieu: "Il y a ceux qui sont faits pour s'emparer des positions faites et ceux qui sont faits pour faire de nouvelles positions. "30 Paiement est de ceux qui doivent se tailler une place à eux.

\section{La récupération du mythe}

Le fantasme d'écrivain tire sa source dans la biographie. Vouloir écrire, dit Barthes, c'est "vouloir copier, non l'œuvre, mais les pratiques, les postures, cette façon de se promener dans le monde, un carnet dans la poche et une phrase dans la tête. ${ }^{31}$. Si le fantasme d'écrivain marque l'aspirant écrivain, il détermine aussi les modes de lecture. Comme le remarque Jean-Louis Major : "Dans la mesure où elle est une institution culturelle, la littérature agit à la façon d'un système mythologique régissant aussi bien les modes de lecture que les formes du discours et les rapports entre l'écrivain et la collectivité. ${ }^{32}$ Toute lecture est déterminée par une prise de position esthétique qui découle d'une conception de la littérature. Comme le souligne Richard SaintGelais: - La gestion préalable de la lecture implique aussi la problématique à partir de laquelle le lecteur aborde le texte: les postulats auxquels il souscrit - le plus souvent tacitement - et, plus

\footnotetext{
30) Ibid., p. 211.

31 Roland Barthes, op. cit., p. 81.

32 Jean-Louis Major, - Perspectives esthétiques: fantasme d'écrivain et figure du texte. op. cit., p. 168 .
} 
généralement, la conception qu'il se fait de son propre exercice. ${ }^{33}$ Le lecteur imprégné d'une conception particulière de l'écrivain et de l'écriture se servira de ses présupposés pour lire le texte. Or, toute lecture fondée sur le fantasme de l'écrivain accordera préséance à la biographie. Certains événements biographiques, le plus souvent tragiques, se prêtent mieux que d'autres à la mythification: la folie, l'exil, la marginalité ou le suicide. Selon Jean-Louis Major, ce "tragique sied bien à un certain fantasme d'écrivain, et le mythe qui s'en alimente oriente la lecture et même en tient lieu. 31 .

Lorsque le fantasme d'écrivain est à l'œuvre, le biographique éclipse le littéraire. Ainsi Claude Abastado remarque qu'^[à] l'époque où le mythe du Poète sacralise l'écrivain, [la critique] découvre la notion d' "auteur" "35. Le but de la critique est alors de comprendre et d'analyser le génie créateur. Dans ce contexte, l'œuvre ne sert qu'à permettre une investigation de la vie de l'auteur. Elle n'est qu'un prétexte pour lire autre chose. Ainsi, tout le discours critique qui porte sur l'œuvre d'André Paiement lie d'emblée et irrémédiablement l'homme et l'œuvre. Dans le théâtre de Paiement, on lit, évidemment, d'abord son suicide. Comme le remarque avec justesse François Paré, "[sla mort aura, bien sûr, largement contribué à sa renommée, d'autant plus qu'elle a été immédiatement prise en charge et sacralisée par plusieurs de ses contemporains et amis, dont les poètes et éditeurs Robert Dickson et Gaston Tremblay "36.

L'homogénéité du discours critique sur l'œuvre d'André Paiement est remarquable. Peut-être est-ce dû au fait que "[l]orsqu'il porte sur un destin individuel, le mythe trace d'ordinaire une démarche exemplaire qui va de la naissance à la mort en dégageant dans l'intervalle des moments ou dés aspects privilégiés reliés de façon significative à l'un ou l'autre des deux pôles. 37 . Toujours est-il que la critique adopte un parcours qui va de la vie à l'œuvre pour rejoindre la communauté. Ce qui étonne d'abord, ce n'est pas le fait que l'on présente la personne de l'auteur - il

\footnotetext{
33 Richard Saint-Gelais, op. cit., p. 86.

34 Jean-Louis Major, * Perspectives esthétiques: fantasme d'écrivain et figure du texte , op. cit., p. 169,

35 Claude Abastado, op. cit., p. 209.

36 François Paré, Thévories de la fragilitê, op. cit., p. 66.

37 Jean-Louis Major, «Saint-Denys Garneau •, Le jeu en étotle, op. cit., p. 86.
} 
s'agit là d'une pratique somme toute courante - c'est plutôt la place prépondérante que l'on fait à sa mort, qui est le plus souvent présentée dans un panégyrique: *Dix ans déjà! Le 23 janvier 1978 mourrait, à Sudbury, André Paiement " ${ }^{38}$; "When André Paiement died in $1978 \ldots$...39; "il meurt subitement et tragiquement le 23 janvier 1978 à Sudbury. Comment expliquer cette disparition à 28 ans?. "10 L'accent est mis sur la mort de Paiement, car l'ensemble du discours sur l'œuvre sera construit à partir de cet événement. L'œuvre de Paiement ne servirait finalement qu'à expliquer sa mort. Le résumé du texte de Paul Gay paru dans Histoire du théâtre au Canada est révélateur à cet égard. Bien que la plus grande partie de l'article soit consacrée à la présentation sous forme de résumé des pièces de Paiement, le sommaire ne fait nullement état de ces dernières. Il souligne au contraire le rapport entre la vie et l'œuvre de Paiement:

Cet article étudie le cas, poussé à l'extrême, d'un FrancoOntarien de Sturgeon-Falls qui s'est enlevé la vie, probablement pour n'avoir jamais admis sa situation de colonisé, et qui a crié - sous le rire et dans le grotesteque des gestes comiques son désir d'indépendance et d'affirmation française dans un milieu anglais. ${ }^{11}$

La conclusion énonce explicitement cette démarche lecturale qui vise d'abord à expliquer la mort (réelle) de l'auteur: ‘L'hypothèse qui sous-tend toutes les pages précédentes pour expliquer la mort tragique d'André Paiement reste plausible. ${ }^{42}$

Le second présupposé qui anime cette forme de critique littéraire est que l'écrivain, sa vie, sa mort, son œuvre symbolisent l'ensemble de la communauté. L'article de Fernand Dorais, "Mais qui a tué André ?" contribue d'autant plus à la mythification d'André Paiement qu'il récupère le biographique à seule fin de l'insérer dans le social. Il n'est d'ailleurs pas étonnant que le nom d'André Paiement n'apparaissent que dans le titre et les derniers

38 Michel Courchesne, - André Paiement. Père de la francophonie ontarienne., Revue du Nouvel-Ontario, $\mathrm{n}^{\circ} 9$, p. 145.

39 Marthe Allain, "Schizophrénic! is what we be" - Or Is it? The FrancoOntarian Heritage in the Plays of André Paiement ", Theatre History in Canada, vol. XI, $\mathrm{n}^{\circ} 1$, printemps 1990 , p. 94.

40 Paul Gay, - Paul-André Paiement (1950-1978), ou le désespoir du colonisé ., Histoire du théatre au Canada, vol. VII, no 2, automne 1986, p. 177.

41 Ibid, p. 176.

42 Ibid, p. 183. 
paragraphes, car il n'est en fait qu'un prétexte, que l'illustration de ce qui est au centre du discours de l'essayiste: les conditions aliénantes de la minorité. Toute la vie de l'écrivain et, par conséquent, toute son œuvre - car la vie explique l'œuvre - trouve son sens dans l'aliénation de la collectivité: *Mais qui a tué André! À la poutre de son logis, il pend, et Pilate de dire: Je m'en lave les mains. L'acculturation et les Franco-Ontariens : hélas! ... „13

Une telle posture lecturale s'inscrit dans une prise de position qui postule que . l'écriture est ici indéniablement traversée par la vie, celle de l'écrivain ayant si tôt mis fin à ses jours et celle de la collectivité minorisée, opprimée, menacée, pour qui la littérature (non pas comme savoir, mais comme action langagière) est peutêtre le signe d'une folle libération. "it: Cette tendance à faire de l'écrivain franco-ontarien, et non seulement de Paiement (qui n'est malheureusement, ici aussi, que le prétexte), "une métaphore de l'existence collective “ ${ }^{45}$ n'est pas exclusive à Dorais: elle est la norme dans la critique littéraire franco-ontarienne. On pourrait croire que ce mode de lecture est, de fait, inscrit dans des textes qui, par leur thématique, appellent une lecture représentative ${ }^{16}$. Or, parler de "décontextualisation " ${ }^{17}$ comme Robert Yergeau ou de "littérature de l'oubli " ${ }^{18}$ comme le fait François Paré, éprouver le besoin de souligner que "l'œuvre de Christensen ne soulève aucune des grandes questions qui ont animé la littérature franco-ontarienne depuis son développement dans les années $70^{4}{ }^{49}$, c'est aussi et toujours adopter une posture lecturale qui trouve son fondement dans le fantasme de l'écrivain minoritaire, en dépit d'œuvres qui s'écrivent et s'inscrivent hors de la problématique identitaire.

Ainsi, dans la critique en société minoritaire, l'auteur et son destin tragique, comme l'œuvre, ne sont en fin de compte que

43 Fernand Dorais: "Mais qui a tué André?., Entre Montréal... et Sudbury, Sudbury, Prise de parole, 1984, p. 28.

44 François Paré, Théories de la fragilité, op. cit., p. 69.

45 Ibid., p. 32.

46 la lecture représentative postule un rapport d'équivalence entre la cliégèse et le référent extratextuel. Voir Lucie Hotte, "L'inscription de la lecture. Romans de la lecture, lecture du roman*, thèse de doctorat, Université d'Ottawa, 1996.

47 Robert Yergeau, *Comment habiter le territoire fictionnel franco-ontarien? Liatson, $\mathrm{n}^{\circ} 85$, janvier 1996 , p. 30-32.

48 François Paré, Les littératures de l'extguité, op. cit., p. 123-135;

49 Id., Théories de la fragilité, op. cit., p. 83. 
des prétextes. La récupération du mythe de l'écrivain par la critique vise moins à glorifier l'écrivain en faisant de lui un saint et un martyr qu'à faire le procès de la société dans laquelle il vit. Le poids de la collectivité détourne la critique du seul auteur pour l'orienter vers un "nous *. Le destin tragique de celui qui écrit ne fait, en somme, que témoigner du destin de tous. Tel serait le mode de lecture imposé par le fantasme de l'écrivain minoritaire.

Les écrivains franco-ontariens font souvent figure d'écrivain maudit. Selon Robert Melançon, être maudit, se dire tel, c'est poser le monde "objectif" comme un ordre qui nous condamne, c'est transposer en destin le malheur d'une subjectivité . ${ }^{50}$. Toutefois, pour les littératures minoritaires, ce n'est pas le malheur d'une subjectivité mais plutôt celui d'une collectivité qui sert de fondement à la malédiction. Comme le souligne François Paré dans Les littératures de l'exiguité, les écrivains qui cherchent à donner la parole à une communauté minoritaire ne peuvent se poser comme singularité, - [c]ar l'écrivain est le porte-parole privilégié de la communauté, touchée par la malédiction. On ne peut donc guère parler d'écrivain maudit, mais de conscience de la malédiction collective " ${ }^{51}$. Ainsi, dans les littératures minoritaires, "le refus du je créateur se fait au nom de la voix collective: par là le nous devient le lieu du système génératif du texte ${ }^{52}$.

Le refus d'être écrivain qui anime André Paiement et ses successeurs, tels Patrice Desbiens ou Pierre Albert, est en réalité un refus de faire partie d'un groupe qui pourrait éventuellement être perçu comme intellectuel, coupé de la collectivité. Mais c'est surtout une façon de prendre ses distances par rapport à un modèle imposé par l'Autre, le majoritaire, celui qui détient le pouvoir hégémonique. En fait, refuser d'être Écrivain, c'est refuser de jouer un rôle préalablement défini : c'est s'affirmer différent. Mais c'est aussi et surtout réitérer, de façon continue et absolue, son appartenance à groupe social exclu des lieux du pouvoir et du savoir. Refuser d'être écrivain et persister à écrire, surtout dans un contexte où l'on croit fermement que *ce qui n'est pas exprimé

50 Robert Melançon, la mott de l'écrivain maudit *, Liberté, vol. Xl, $\mathrm{n}^{\mathrm{N}}$ 3-4, 1969 , p. 25.

51 François Paré, Les littératures de l'exiguité, op. cit., p. 130.

52 Liliàn Pestre de Almeida, * Regard périphérique sur la francophonie ou pourquoi et comment enseigner les littératures francophones dans les Amétiques -, Études littératres, vol. XVI, $\mathrm{n}^{\circ} 2$, août 1983, p. 264. 
n'existe pas. ${ }^{53}$ et qu'il faut prendre la parole pour faire advenir la réalité, c'est se condamner à une lutte sans fin avec le fantasme d'écrivain. Je ne peux que reprendre ici, en souhaitant que cette posture devienne finalement celle de la critique littéraire francoontarienne, la définition que Jean-Louis Major donne du * parcours d'une lecture qui se veut authentiquement littéraire: rencontre, toujours au présent, de l'écriture et du lecteur, en quoi se révèle et se renouvelle, par-delà le fantasme d'écrivain, la figure, unique, inaltérable, du texte. C'est à cette condition que continuent ou, faudrait-il dire, que commencent d'exister les ouvres. 51 .

53 Gaston Tremblay, op. cit., p. 71.

54 Jean-Louis Major, "Perspectives esthétiques: fantasme d'écrivain et figure du texte *, op. cit., p. 173-174. 